\title{
Construcción y validación de un instrumento para caracterizar el nivel de innovación en instituciones prestadoras de servicios de salud
}

\author{
ChaVARria ChaVARria, TATIANA MARgarita \\ Facultad de Administración. Universidad CES (Colombia) \\ Correo electrónico: tchavarria@ces.edu.co \\ PUlgarín MOLINA, SERGIO ANDRÉS \\ Escuela de Administración. Universidad del Rosario (Colombia) \\ Correo electrónico: sergio.pulgarin@urosario.edu.co
}

\begin{abstract}
RESUMEN
La investigación pretende desarrollar y validar un instrumento para caracterizar el nivel de impacto, en las instituciones prestadoras de servicios de salud, de la aplicación de las cuatro dimensiones esenciales del Radar de la innovación: las ofertas de la institución (¿qué?), los clientes que atiende (¿a quién?), los procesos que utiliza (¿cómo?) y los puntos de presencia que emplea para llevar sus ofertas al mercado (¿dónde?).

Se optó por un estudio de corte exploratorio mixto. Mediante la revisión bibliográfica y la discusión con un panel de 11 expertos, se construyó y aprobó el contenido del instrumento de 49 ítems en escala Likert de 5 puntos para caracterizar la utilización de la innovación en las instituciones prestadoras de servicios de salud; el constructo y la fiabilidad fueron validados mediante la aplicación de una prueba piloto a 33 participantes y el estudio de los resultados obtenidos para cada una de las dimensiones del Radar de la innovación se realizó empleando el Análisis Factorial Exploratorio (AFE) y el alfa de Cronbach.

La investigación propone un instrumento cuyo contenido y cuyo constructo fueron validados para la estructura factorial final compuesta por 43 ítems incluidos en 13 factores o criterios; mediante el análisis estadístico se evidencia alta fiabilidad para las cuatro dimensiones que propone el Radar de la innovación.
\end{abstract}

Palabras clave: innovación, radar de la innovación, innovación en salud, instituciones prestadoras de servicios de salud, validación de instrumentos de medición.

Clasificación JEL: O32; I10.

MSC2010: 90B50; 62H25.

Artículo recibido el 22 de agosto de 2018 y aceptado el 1 de julio de 2019. 


\title{
Construction and validation of an instrument to characterize the level of innovation in healthcare institutions
}

\begin{abstract}
The research aims to develop and validate an instrument to characterize the impact level, in the healthcare institutions, of the application of the four essential dimensions of the Radar of innovation: the offers of the institution (what?), the clients (who?), the processes (how?) and the points of presence (where?).

A mixed exploratory study was chosen. Through the bibliographic review and the discussion with a panel of 11 experts, the content of the instrument of 49 items was constructed and approved on a 5-point Likert scale to characterize the use of innovation in the healthcare institutions; the construct and reliability were validated by applying a pilot test to 33 participants and the study of the results obtained for each of the dimensions of the innovation radar was carried out using exploratory factor analysis (AFE) and Cronbach's alpha.

The research proposes an instrument whose content and construct were validated for the final factorial structure composed of 43 items, included in 13 factors or criteria; through statistical analysis, high reliability is demonstrated for the four dimensions proposed by the Radar of innovation.
\end{abstract}

Keywords: innovation, innovation radar, innovation in health, healthcare institutions, validation instruments of measure.

JEL classification: O32; I10.

MSC2010: 90B50; 62H 25 .

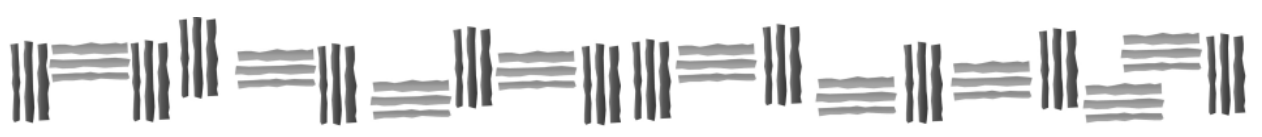




\section{Introducción.}

La innovación surge como una alternativa de transformación para los cambios en el entorno y como una opción para la generación de ventajas competitivas en las empresas. Sin embargo, los esfuerzos realizados en salud no tienen sentido si desde el punto de vista del paciente no se perciben beneficios relacionados con una mejor salud y para la organización un aumento en la eficiencia de las operaciones internas y la calidad en la atención (Länsisalmi, Kivimäki, Aalto \& Ruoranen, 2006).

Para generar evidencias que permitan a los usuarios y al sistema de salud percibir los beneficios de la innovación, se requiere caracterizar su impacto en la calidad de la atención, la seguridad del paciente y la reducción de costos innecesarios en la prestación de los servicios (Giacometti-Rojas, 2013; Organización Panamericana de la Salud [OPS], 2009).

El Manual de Oslo clasifica la innovación en cuatro tipos de acuerdo al objetivo buscado: innovación en producto, innovación en mercado, innovación en proceso e innovación en la organización (Organización para la Cooperación y el Desarrollo Económicos [OCDE], 2005); por su parte Barba (2012) manifiesta que existen 10 tipos de innovación de acuerdo a los expuestos por Doblin para los ámbitos estratégicos y los campos en los que es posible innovar en las organizaciones.

Para efectos de este estudio se utilizó un método de clasificación de innovación integrado denominado Radar de la innovación, propuesto por Sawhney, Wolcott y Arroniz (2007) que realiza una aproximación hacia la caracterización de la innovación mediante cuatro dimensiones esenciales: las ofertas de la empresa (¿qué?), los clientes que atiende (¿a quién?), los procesos que utiliza (¿cómo?) y los puntos de presencia que emplea para llevar sus ofertas al mercado (¿dónde?); en cada uno de estos cuatro puntos de referencia, el método incorpora 3 criterios para un total de 12 factores en los que las empresas pueden innovar.

Para la dimensión relacionada con los productos, servicios o el qué ofertado por la empresa, los criterios específicos en los que se puede innovar son: oferta, plataforma y soluciones; para la dimensión relacionada con el consumidor, usuario o a quién va dirigida la oferta, los criterios específicos son: clientes, experiencia del cliente y obtención de valor; en la dimensión de los procesos o el cómo, los criterios son: procesos, organización y cadena de suministro y, finalmente, para la dimensión presencia o en dónde, los criterios propuestos son presencia, conexiones y marca (Sawhney et al., 2007).

En esta investigación se desarrolla y valida un instrumento para la medición del impacto, en las instituciones prestadoras de servicios de salud, de los 12 criterios o factores de la innovación propuestos por Sawhney et al. (2007); el contenido del instrumento de 49 ítems tipo escala Likert fue aprobado por un panel de juicio de expertos y el constructo y la fiabilidad se validaron por medio de una prueba piloto cuyos resultados se analizaron mediante técnicas estadísticas como el Análisis Factorial Exploratorio (AFE) y el alfa de Cronbach.

Se obtiene un instrumento simplificado que representa las cuatro dimensiones propuestas por el Radar de la innovación, con 13 factores o criterios en los que las instituciones pueden innovar (uno más que el instrumento original) y un total de 43 criterios (6 menos que el instrumento original).

El instrumento desarrollado permite, a las instituciones prestadoras de servicios de salud, caracterizar el rol de la innovación de manera rápida, de bajo costo y teniendo en cuenta todas las áreas y procesos de las entidades.

\section{Metodología.}

Esta investigación se enmarca en un diseño exploratorio mixto que parte de la revisión de la literatura y la observación. Para recoger los datos analizados, se llevó a cabo un estudio de carácter no 
experimental de tipo transeccional, dado que el trabajo de campo fue realizado en un momento específico, con el fin de identificar el estado de la variable de estudio, en este caso, la innovación en el sector salud (Hernández, Fernandez \& Baptista, 2010).

A continuación, se describe la manera en la que se llevó a cabo el estudio en términos de número y características de los participantes, el instrumento desarrollado y empleado para la caracterización de la innovación en salud y el procedimiento empleado en el marco de ésta.

\section{Participantes}

Para la etapa de construcción y aprobación del contenido del instrumento, se conformó un panel de discusión con 11 expertos que tenían el siguiente perfil: 6 docentes e investigadores de instituciones de educación superior y 5 coordinadores de las áreas de Investigación, Desarrollo e Innovación (I+D+i) de instituciones prestadoras de servicios de salud.

Se seleccionó el panel de expertos teniendo en cuenta las recomendaciones de Hyrkäs, Appelqvist-Schmidlechner y Oksa (2003) para quienes un panel de expertos, conformado por mínimo diez participantes, brinda una estimación confiable de la validez del instrumento.

En la prueba piloto, cuya finalidad fue validar el constructo y fiabilidad del instrumento, participaron voluntariamente 33 expertos en innovación en salud de la ciudad de Medellín, con la siguiente distribución de acuerdo al área de trabajo o al tipo de actividad realizada: 11 docentes e investigadores universitarios, 7 expertos del área de infraestructura y tecnologías hospitalarias, 7 expertos de áreas directamente relacionadas con la atención en salud y la prestación de servicios, 6 pertenecían al área comercial y de comunicaciones y 2 al área de planes y proyectos en salud.

Se seleccionó el tamaño de la muestra para la prueba piloto teniendo en cuenta las recomendaciones de Babbie (2000) que manifiesta que el tamaño de muestra para estudios piloto debe ser entre 30 y 50 participantes y todos los expertos deben poseer los atributos que se desean medir en la población objetivo.

Los atributos que se tuvieron en cuenta para la selección de los participantes de la prueba piloto fueron los recomendados por Skjong y Wentworth (2000): experiencia en el sector salud, experiencia y conocimientos en innovación, alta reputación en la comunidad, disponibilidad para participar e imparcialidad.

\section{Instrumento}

Se desarrolló un instrumento, en adelante HCI (Herramienta de Caracterización de la Innovación), basado en la metodología propuesta por Sawhney et al. (2007) en el Radar de la innovación.

De cara a la innovación en salud, se realizó una revisión bibliográfica y se tuvieron en cuenta los siguientes autores: del Llano, Marténez-Cantarero, Gol y Raigada (2002); Djellal y Gallouj (2005); Guan, Yam, Mok y Ma (2006); Jaramillo, Latorre, Albán y Lopera (2008); Keeley, Pikkel, Quinn y Walters (2013); Palencia-Sánchez y García-Ubaque (2016); Thune y Mina (2016); y con el apoyo del panel de expertos se definieron los ítems a evaluar en cada dimensión para conformar la HCI empleando el método propuesto por Sawhney et al. (2007).

Se seleccionaron 49 ítems para las cuatro dimensiones del Radar de la innovación, cada uno representado de la siguiente manera; para la dimensión ¿qué? un total de 11 ítems; para la dimensión ¿a quién? un total de 14 ítems; en la dimensión ¿cómo? se incluyeron 13 ítems y, finalmente, para la dimensión ¿dónde? un total de 11 ítems. 
Los ítems de la HCI se evalúan a través de una escala tipo Likert, donde 1 es "muy en desacuerdo" y 5 es "muy de acuerdo", permitiendo así medir la percepción y el grado de conformidad del experto con cada una de las afirmaciones propuestas para los criterios del Radar de la innovación.

\section{Procedimiento}

A continuación, se detallan cada una de las etapas del proceso de validación del instrumento.

a) Aprobación del contenido del instrumento diseñado.

La aprobación del contenido de un instrumento no puede expresarse cuantitativamente, se estima de manera subjetiva empleando para este caso el juicio de los expertos. Se recurre a este método para conocer la probabilidad de error en la configuración del instrumento (Corral, 2009).

Para aprobar el contenido de la HCI, conforme a lo planteado por Hyrkäs et al. (2003), en esta investigación se consideró que un ítem sería incorporado si el 80\% de los expertos estaban de acuerdo con su validez.

Para evaluar cualitativamente el instrumento, el panel de expertos calificó, positiva o negativamente, cada uno de los ítems incluidos en la HCI para los siguientes criterios: claridad en la redacción, inducción a la respuesta, lenguaje adecuado y percepción de que los ítems realmente miden lo que se pretende.

Este proceso permitió evaluar el instrumento a partir de su claridad desde el punto de vista del significado y la formulación, efectuando las modificaciones que se consideraban oportunas para garantizar la buena redacción y, por consiguiente, brevedad y facilidad de comprensión.

b) Validez del constructo.

Entre las técnicas estadísticas más utilizadas para validar el constructo de un instrumento de medición, se destacan las dos modalidades del análisis factorial (AF): el análisis factorial exploratorio (AFE) y el análisis factorial confirmatorio (AFC); la diferencia principal entre ambos análisis radica en que el AFE es un procedimiento descriptivo que busca definir los grupos de variables o factores que están altamente correlacionados entre sí, mientras que el AFC se usa cuando el problema de investigación está vinculado a una teoría que se ha probado anteriormente (Detrinidad, 2016; Pérez-Gil, Chacón \& Moreno, 2000).

Para este estudio, se utilizó el AFE ya que el objetivo fue determinar si los ítems estaban correctamente agrupados en cada uno de los criterios en los que se subdividen las cuatro dimensiones del Radar de la innovación e igualmente, describir los factores y determinar si los propuestos eran suficientes para explicar los resultados que proporcionan todas las preguntas del instrumento.

Para esta etapa del estudio, se aplicó una prueba piloto a 33 expertos, quienes evaluaron los 49 ítems incluidos en la HCI de escala Likert de acuerdo a la percepción del impacto considerado para el criterio y la dimensión que los contenían.

Los expertos fueron contactados a través de correos electrónicos institucionales, se inició con la socialización y explicación de la HCI para posteriormente realizar la evaluación.

El análisis factorial se realizó empleando el programa estadístico SPSS Statistics versión 23, inicialmente se calculó la medida de adecuación muestral de Kaiser, Meyer y Olkin (KMO) y el valor del nivel de significación de la prueba de esfericidad de Barlett para cada una de las dimensiones; se aprobó la realización del AFE si el KMO era superior a 0,5 y el nivel de significación de Barlett inferior a 0,05 para cada una de las dimensiones de la HCI (Montoya, 2007). 
Para extraer los factores se suelen utilizar dos métodos estadísticos: el análisis de componentes principales (ACP) y el análisis de ejes principales (AEP). Para esta investigación se ha usado el ACP debido a que al analizar la varianza total, este método permite obtener los mejores resultados con la menor perdida de información, ya que el ACP sugiere extraer únicamente los autovalores cuyo valor es superior a 1; sin embargo, al emplear este método estadístico, el principal problema radica en que los resultados en bruto son difíciles de analizar por lo que se aplica la rotación ortogonal u oblicua para generar una estructura simple (Pérez \& Medrano, 2010; Zamora, Monroy \& Chávez, 2009).

Para esta investigación se aplica la rotación ortogonal tipo Varimax, que facilita la interpretación debido a que se genera una estructura factorial simple cuyas variables están altamente cargadas en un solo factor (Zamora et al., 2009).

c) Análisis de fiabilidad.

Para demostrar que los ítems del instrumento tienen consistencia interna, se desarrolló un análisis de fiabilidad. Éste se refiere al grado en que su aplicación repetida al mismo sujeto u objeto, produce iguales resultados (Hernández et al., 2010).

El índice de fiabilidad para cada una de las dimensiones del instrumento se obtiene mediante el cálculo del estadístico alfa de Cronbach $\alpha$ utilizando la Ecuación 1.

$$
\alpha=\left(\frac{K}{K-1}\right) \times\left(1-\frac{\sum_{i=1}^{k} S i^{2}}{S t^{2}}\right)
$$

donde:

$K$ es el número de ítems (preguntas) del cuestionario

$S i^{2}$ Corresponde a la varianza de cada ítem

$S t^{2}$ Indica la varianza total del cuestionario (Suma de varianza de los evaluadores).

Ecuación 1. Cálculo del estadistico alfa de Cronbach.

La literatura especializada no presenta valores estándar para la interpretación del alfa de Cronbach; algunos autores aseguran que los aceptados se encuentran entre 0,6 y 0,8 en análisis exploratorios o primeras fases de la investigación (Huh, DeLorme, \& Reid, 2006; Jaramillo \& Osses, 2012), otros investigadores expresan que valores de coeficientes confiables oscilan entre 0,7 y 0,9 (Campo-Arias \& Oviedo, 2008; Kaplan, Saccuzzo \& Reyes, 2006).

Por otro lado, Ruiz (1998) propone una manera para establecer los rangos de magnitud del coeficiente de acuerdo a la escala presentada en la Tabla 1, que se empleó en este estudio para el análisis de los resultados.

Tabla 1. Rangos de valoración de confiabilidad mediante alfa de Cronbach.

\begin{tabular}{|c|c|}
\hline Rangos & Magnitud \\
\hline 0,81 a 1,00 & Muy alta \\
\hline 0,61 a 0,80 & Alta \\
\hline 0,40 a 0,60 & Moderada \\
\hline 0,21 a 0,40 & Baja \\
\hline 0,01 a 0,20 & Muy Baja \\
\hline
\end{tabular}

Fuente: Ruiz (1998). 


\section{Resultados.}

A continuación, se presentan los resultados de la validación de la HCI; en primer lugar, se presentará el análisis de la validez del contenido del instrumento inicial empleando el panel de juicio de expertos, posteriormente se muestran los resultados de la validez del constructo y la fiabilidad del instrumento para, finalmente, proponer ajustes a la HCI de acuerdo al análisis estadístico.

\subsection{Validación del contenido del instrumento.}

Los 11 participantes que conformaron el panel de juicio de expertos, coincidieron en la importancia de aplicar el instrumento dividido en las dimensiones (¿qué?, ¿a quién?, ¿cómo? y ¿dónde?) y estuvieron de acuerdo con los 49 ítems tipo escala Likert desarrollados.

En la dimensión “¿dónde?”, algunos de los ítems incluidos en los criterios "presencia” y "conexiones" fueron formulados de tal manera que inducían sesgo por lo que los expertos sugirieron ajustar la redacción.

La Tabla 2 presenta el contenido de la HCI con los 49 ítems inicialmente definidos y teniendo en cuenta las recomendaciones de los expertos en la validación del contenido de la herramienta.

Tabla 2. Ítems incluidos en la primera versión de la HCI.

\begin{tabular}{|c|}
\hline DIMENSIÓN ¿QUÉ? \\
\hline OFERTA \\
\hline Q3 Apertura de servicios. \\
\hline Q4 Mejoramiento de los servicios existentes. \\
\hline Q5 Facilidad de acceso y personalización de la oferta. \\
\hline $\begin{array}{l}\text { Q6 Generación de paquetes de servicios y productos (varios servicios y productos relacionados y su venta } \\
\text { en un solo paquete). }\end{array}$ \\
\hline PLATAFORMA \\
\hline Q7 Dotación y aprovechamiento de la tecnología biomédica. \\
\hline Q8 Aprovechamiento de la infraestructura física. \\
\hline Q9 Actividades de docencia e investigación. \\
\hline Q10 Suministro de diferentes líneas de productos y servicios (incluyendo líneas complementarias). \\
\hline SOLUCIONES \\
\hline Q11 Generación de asociaciones y alianzas. \\
\hline Q12 Integración del portafolio (incluyendo servicios sanitarios y no sanitarios). \\
\hline Q13 Identificación del perfil de los clientes para brindar mejores soluciones. \\
\hline DIMENSIÓN ¿A QUIÉN? \\
\hline CLIENTES \\
\hline Q14 Relación entre la calidad y el precio. \\
\hline Q15 Segmentación de los clientes. \\
\hline Q16 Nuevos nichos de mercado (identificación de necesidades insatisfechas) \\
\hline
\end{tabular}




\section{EXPERIENCIA DEL CLIENTE}

Q17 Atención e información al cliente.

Q18 Calidad y seguridad en la prestación de los servicios.

Q19 Participación y pertenencia del usuario y sus acompañantes en todo el proceso de prestación de servicios.

Q20 Servicios de preventa y posventa.

Q21 Medios de atención

\section{OBTENCIÓN DE VALOR}

Q22 Actividades comerciales e industriales diferentes a la prestación de servicios de salud.

Q23 Participación en proyectos de impulso del sector.

Q24 Publicidad.

Q25 Formas de pago o financiación.

Q26 Interacción con proveedores, competidores y otros aliados.

Q27 Participación en redes y trabajo colaborativo.

DIMENSIÓN ¿CÓMO?

\section{PROCESOS}

Q28 Sistemas de información e informática

Q29 Implementación y uso de estándares nacionales e internacionales.

Q30 Logística de la prestación de los servicios.

\section{ORGANIZACIÓN}

Q31 Integración de la tecnología biomédica con las tecnologías de la información y la comunicación.

Q32 Profesionales y especialistas en salud (talento humano).

Q33 Educación continua y formación.

Q34 Sistemas de incentivos.

Q35 Gestión del conocimiento

\section{CADENA DE SUMINISTRO}

Q36 Integración de la cadena de suministro.

Q37 Generación de alianzas estratégicas.

Q38 Implementación y uso de TI (Tecnologías de la información).

Q39 Logística en la cadena de suministro.

Q40 Gestión de la información.

\section{DIMENSIÓN ¿DÓNDE?}

\section{PRESENCIA}

Q41 Generación de alianzas estratégicas para afianzar la presencia.

Q42 Ubicación.

Q43 Diversificación.

Q44 Cobertura y atención internacional.

\section{CONEXIONES}

Q45 Relación con otros miembros de la red de prestadores de servicios.

Q46 Implementación y uso de medios de atención a distancia. 
Q47 Implementación de alianzas estratégicas y de colaboración.

Q48 Confidencialidad de la información y confiabilidad de la institución.

\section{MARCA}

Q49 Extensión de la marca a otros campos

Q50 Reconocimiento nacional e internacional de la marca.

Q51 Percepción, posición y aceptación de la marca vs la competencia.

Fuente: Elaboración propia.

La Tabla 3 presenta los resultados de la aprobación del contenido del instrumento en cuanto al porcentaje de expertos que manifestaron que los ítems eran claros, empleaban el lenguaje adecuado, median la innovación en salud y en la redacción no se inducía la respuesta.

Tabla 3. Resultados de la medición para la aprobación del contenido del instrumento.

\begin{tabular}{|c|c|c|c|c|}
\hline \multirow{2}{*}{$\begin{array}{l}\text { CRITERIOS } \\
\text { DEL RADAR DE } \\
\text { LA } \\
\text { INNOVACIÓN }\end{array}$} & \multicolumn{4}{|c|}{ CRITERIOS A EVALUAR } \\
\hline & Redacción & $\begin{array}{l}\text { Inducción a la } \\
\text { respuesta } \\
\text { (sesgo) }\end{array}$ & $\begin{array}{l}\text { Lenguaje } \\
\text { adecuado }\end{array}$ & $\begin{array}{l}\text { Los criterios en general } \\
\text { miden lo que se pretende }\end{array}$ \\
\hline \multicolumn{5}{|c|}{ Dimensión: ¿Qué? } \\
\hline Oferta & $90,9 \%$ & $0 \%$ & $100 \%$ & $100 \%$ \\
\hline Plataforma & $90,9 \%$ & $9,09 \%$ & $100 \%$ & $100 \%$ \\
\hline Soluciones & $100 \%$ & $9,09 \%$ & $100 \%$ & $90,9 \%$ \\
\hline \multicolumn{5}{|c|}{ Dimensión: ¿A quién? } \\
\hline Clientes & $81,81 \%$ & $0 \%$ & $100 \%$ & $100 \%$ \\
\hline $\begin{array}{l}\text { Experiencia del } \\
\text { cliente }\end{array}$ & $100 \%$ & $0 \%$ & $100 \%$ & $90,9 \%$ \\
\hline $\begin{array}{l}\text { Obtención de } \\
\text { valor }\end{array}$ & $100 \%$ & $9,09 \%$ & $100 \%$ & $100 \%$ \\
\hline \multicolumn{5}{|c|}{ Dimensión ¿Cómo? } \\
\hline Procesos & $90,9 \%$ & $0 \%$ & $100 \%$ & $90,9 \%$ \\
\hline Organización & $100 \%$ & $0 \%$ & $100 \%$ & $90,9 \%$ \\
\hline $\begin{array}{ll}\text { Cadena de } \\
\text { suministro }\end{array}$ & $90,9 \%$ & $0 \%$ & $90,9 \%$ & $100 \%$ \\
\hline \multicolumn{5}{|c|}{ Dimensión: ¿Dónde? } \\
\hline Presencia & $100 \%$ & $72,72 \%$ & $100 \%$ & $100 \%$ \\
\hline Conexiones & $90,9 \%$ & $63,63 \%$ & $100 \%$ & $100 \%$ \\
\hline Marca & $90,9 \%$ & $0 \%$ & $100 \%$ & $100 \%$ \\
\hline
\end{tabular}

Fuente: Elaboración propia. 


\subsection{Validez del constructo y fiabilidad.}

Se aplicó el AFE y se calculó el coeficiente alfa de Cronbach para validar el constructo y la fiabilidad de cada una de las dimensiones del Radar de la innovación. A continuación, se presentan los resultados de este análisis.

\section{AFE y fiabilidad para la dimensión "¿qué?"}

Inicialmente se validó la aplicabilidad del AFE ya que se encontró un KMO entre 0,5 y 1 ; y la prueba de esfericidad de Barlett arrojó un resultado menor a 0,05 (Montoya, 2007).

La Tabla 4 presenta los autovalores iniciales para la dimensión “¿qué?”: se definen cuatro factores de extracción debido a que tienen valores mayores a 1 y explican el 70,83\% de la varianza total de los datos originales.

Tabla 4. Autovalores iniciales para la dimensión “¿qué?”.

\begin{tabular}{|l|l|l|l|}
\hline \multirow{2}{*}{ Ítem } & \multicolumn{3}{|c|}{ Autovalores iniciales } \\
\cline { 2 - 4 } & Total & \% de varianza & \% acumulado \\
\hline 1 & $\mathbf{4 , 3 4 8}$ & 39,523 & 39,523 \\
\hline 2 & $\mathbf{1 , 2 9 7}$ & 11,791 & 51,315 \\
\hline 3 & $\mathbf{1 , 1 2 4}$ & 10,215 & 61,529 \\
\hline 4 & $\mathbf{1 , 0 2 3}$ & 9,296 & $\mathbf{7 0 , 8 2 5}$ \\
\hline 5 & 0,836 & 7,604 & 78,430 \\
\hline 6 & 0,650 & 5,906 & 84,336 \\
\hline 7 & 0,502 & 4,566 & 88,902 \\
\hline 8 & 0,427 & 3,885 & 92,787 \\
\hline 9 & 0,415 & 3,771 & 96,558 \\
\hline 10 & 0,249 & 2,268 & 98,826 \\
\hline 11 & 0,129 & 1,174 & 100,000 \\
\hline
\end{tabular}

Sin embargo, inicialmente se habían planteado de acuerdo con el Radar de la innovación, 3 criterios o factores que son: oferta, plataforma y soluciones; por esto se decide aplicar el análisis de fiabilidad para determinar la posible eliminación de algún ítem y, por ende, de algún factor.

El alfa de Cronbach para esta dimensión fue de 0,75 pero este valor aumenta a 0,77 al eliminar el ítem Q6.

La Tabla 5 presenta los resultados de pruebas KMO y esfericidad de Barlett que confirman nuevamente la aplicabilidad del AFE; con el instrumento dimensional modificado y aplicando la rotación Varimax continúan los cuatro factores que explican el 74,30\% de la varianza total de los datos y la distribución de los ítems se presenta en la Tabla 5. 
Tabla 5. Resultados AFE instrumento modificado para la dimensión "¿qué?".

\begin{tabular}{|c|c|c|c|c|c|}
\hline KMO & \multicolumn{5}{|l|}{0,59} \\
\hline Esfericidad de Barlett & \multicolumn{5}{|l|}{0,00} \\
\hline \multicolumn{6}{|l|}{ Matriz de factores } \\
\hline & & \multicolumn{4}{|c|}{ Factores } \\
\hline & & 1 & 2 & 3 & 4 \\
\hline Q4 Mejoramiento de los & existentes. & 0,772 & & & \\
\hline $\begin{array}{l}\text { Q5 Facilidad de acceso } \\
\text { oferta. }\end{array}$ & zación de la & 0,684 & & & \\
\hline $\begin{array}{l}\text { Q12 Integración del port } \\
\text { servicios sanitarios y no san }\end{array}$ & luyendo & 0,749 & & & \\
\hline $\begin{array}{l}\text { Q13 Identificación del p } \\
\text { brindar mejores soluciones. }\end{array}$ & clientes para & 0,803 & & & \\
\hline Q9 Actividades de doce & stigación. & & 0,870 & & \\
\hline $\begin{array}{l}\text { Q10 Suministro de difer } \\
\text { y servicios (incluyendo líne }\end{array}$ & $\begin{array}{l}\text { s de productos } \\
\text { nentarias). }\end{array}$ & & 0,639 & & \\
\hline $\begin{array}{l}\text { Q7 Dotación y aprovech } \\
\text { biomédica. }\end{array}$ & la tecnología & & & 0,799 & \\
\hline Q8 Aprovechamiento de & tructura física. & & & $\mathbf{0 , 7 5 5}$ & \\
\hline Q3 Apertura de servicio & & & & & 0,799 \\
\hline Q11 Generación de asoc & alianzas. & & & & $\mathbf{0 , 8 0 3}$ \\
\hline
\end{tabular}

Fuente: Elaboración propia.

\section{AFE y fiabilidad para la dimensión "¿a quién?"}

Inicialmente se validó la aplicabilidad del AFE ya que se encontró un KMO entre 0,5 y 1 ; y la prueba de esfericidad de Barlett arrojó un resultado menor a 0,05 (Montoya, 2007).

La Tabla 6 presenta los autovalores iniciales para la dimensión “¿a quién?”; se definen cuatro factores de extracción puesto que tienen valores mayores a 1 y explican el $70 \%$ de la varianza total de los datos originales.

Sin embargo, inicialmente se habían planteado de acuerdo con el Radar de la innovación, 3 criterios o factores que son: clientes, experiencia del cliente y obtención de valor; por esto se decide aplicar el análisis de fiabilidad para determinar si se hace necesaria la eliminación de algún ítem y, por ende, de algún factor.

El alfa de cronbach para esta dimensión fue de 0,82 pero este valor aumenta a 0,85 al eliminar los ítems Q22 y Q25. 
Tabla 6. Autovalores iniciales para la dimensión "¿a quién?".

\begin{tabular}{|c|c|c|c|}
\hline \multirow[t]{2}{*}{ Ítem } & \multicolumn{3}{|c|}{ Autovalores iniciales } \\
\hline & Total & $\%$ de varianza & $\%$ acumulado \\
\hline 1 & 4,747 & 33,906 & 33,906 \\
\hline 2 & 1,977 & 14,121 & 48,027 \\
\hline 3 & 1,721 & 12,295 & 60,321 \\
\hline 4 & 1,353 & 9,664 & 69,985 \\
\hline 5 & 0,979 & 6,990 & 76,975 \\
\hline 6 & 0,728 & 5,198 & 82,173 \\
\hline 7 & 0,604 & 4,312 & 86,485 \\
\hline 8 & 0,472 & 3,371 & 89,856 \\
\hline 9 & 0,344 & 2,454 & 92,309 \\
\hline 10 & 0,334 & 2,385 & 94,694 \\
\hline 11 & 0,297 & 2,118 & 96,812 \\
\hline 12 & 0,209 & 1,492 & 98,304 \\
\hline 13 & 0,144 & 1,029 & 99,332 \\
\hline 14 & 0,093 & 0,668 & 100,000 \\
\hline
\end{tabular}

Fuente: Elaboración propia.

La Tabla 7 presenta los resultados de pruebas KMO y esfericidad de Barlett que confirman nuevamente la aplicabilidad del AFE; con el instrumento dimensional modificado y aplicando la rotación Varimax continúan los cuatro factores que explican el 74,61\% de la varianza total de los datos y la distribución de los ítems se presenta en la Tabla 7.

Tabla 7. Resultados AFE instrumento modificado para la dimensión "¿a quién?".

\begin{tabular}{|c|c|c|c|c|}
\hline KMO & \multicolumn{3}{|c|}{0,71} & \\
\hline Esfericidad de Barlett & \multicolumn{3}{|c|}{0,00} & \\
\hline \multicolumn{5}{|c|}{ Matriz de factores rotada } \\
\hline & \multicolumn{4}{|c|}{ Factores } \\
\hline & 1 & 2 & 3 & 4 \\
\hline Q17 Atención e información al cliente. & 0,774 & & & \\
\hline $\begin{array}{l}\text { Q18 Calidad y seguridad en la prestación de los } \\
\text { servicios. }\end{array}$ & $\mathbf{0 , 5 9 0}$ & & & \\
\hline $\begin{array}{l}\text { Q19 Participación y pertenencia del usuario y sus } \\
\text { acompañantes en todo el proceso de prestación de } \\
\text { servicios. }\end{array}$ & $\mathbf{0 , 7 8 9}$ & & & \\
\hline Q20 Servicios de preventa y posventa. & 0,761 & & & \\
\hline
\end{tabular}




\begin{tabular}{|c|c|c|c|}
\hline Q14 Relación entre la calidad y el precio. & $\mathbf{0 , 8 2 3}$ & & \\
\hline Q15 Segmentación de los clientes. & 0,616 & & \\
\hline $\begin{array}{l}\text { Q16 Nuevos nichos de mercado (identificación de } \\
\text { necesidades insatisfechas) }\end{array}$ & $\mathbf{0 , 5 5 7}$ & & \\
\hline Q24 Publicidad. & 0.852 & & \\
\hline Q21Medios de atención. & & 0,701 & \\
\hline $\begin{array}{l}\text { Q26 Interacción con proveedores, competidores y otros } \\
\text { aliados. }\end{array}$ & & $\mathbf{0 , 8 5 4}$ & \\
\hline Q23 Participación en proyectos de impulso del sector. & & & $\mathbf{0 , 8 5 1}$ \\
\hline Q27 Participación en redes y trabajo colaborativo. & & & 0.775 \\
\hline
\end{tabular}

Fuente: Elaboración propia.

\section{AFE y fiabilidad para la dimensión "¿cómo?”.}

Inicialmente se validó la aplicabilidad del AFE ya que se encontró un KMO entre 0,5 y 1 ; y la prueba de esfericidad de Barlett arrojó un resultado menor a 0,05 (Montoya, 2007).

La Tabla 8 presenta los autovalores iniciales para la dimensión “¿cómo?”, se definen cuatro factores de extracción pues tienen valores mayores a 1 y explican el $68,47 \%$ de la varianza total de los datos originales.

Tabla 8. Autovalores iniciales para la dimensión "¿cómo?".

\begin{tabular}{|l|l|l|l|}
\hline \multirow{2}{*}{ Ítem } & \multicolumn{3}{|c|}{ Autovalores iniciales } \\
\cline { 2 - 4 } & Total & \% de varianza & \% acumulado \\
\hline 1 & $\mathbf{5 , 6 5 5}$ & 43,502 & 43,502 \\
\hline 2 & $\mathbf{2 , 0 3 9}$ & 15,682 & 59,184 \\
\hline 3 & $\mathbf{1 , 2 0 7}$ & 9,286 & $\mathbf{6 8 , 4 7 0}$ \\
\hline 4 & 0,941 & 7,241 & 75,711 \\
\hline 5 & 0,809 & 6,224 & 81,936 \\
\hline 6 & 0,634 & 4,880 & 86,816 \\
\hline 7 & 0,586 & 4,506 & 91,322 \\
\hline 8 & 0,350 & 2,694 & 94,016 \\
\hline 9 & 0,268 & 2,060 & 96,076 \\
\hline 10 & 0,207 & 1,592 & 97,668 \\
\hline 11 & 0,153 & 1,180 & 98,848 \\
\hline 12 & 0,105 & 0,811 & 100,000 \\
\hline 13 & 0,044 & 0,341 & \\
\hline
\end{tabular}

Fuente: Elaboración propia. 
El Radar de la innovación para esta dimensión propone tres criterios o factores que son: procesos, organización y cadena de suministro; por esto se decide aplicar el análisis de fiabilidad para determinar la consistencia interna del instrumento y evaluar si se requieren cambios en los ítems.

El alfa de Cronbach para esta dimensión fue de 0,88 que se considera "muy alto" y teniendo en cuenta que los tres factores explican adecuadamente la varianza total, no se eliminan ítems para esta categoría.

La Tabla 9 presenta los resultados de pruebas KMO y esfericidad de Barlett que confirman la aplicabilidad del AFE; con el instrumento dimensional modificado y aplicando la rotación Varimax se tienen tres factores con la distribución de los ítems que se ve en la Tabla 9.

Tabla 9. Resultados AFE instrumento modificado para la dimensión "¿cómo?".

\begin{tabular}{|c|c|c|c|}
\hline KMO & \multicolumn{3}{|c|}{0,63} \\
\hline Esfericidad de Barlett & \multicolumn{3}{|c|}{0,00} \\
\hline \multicolumn{4}{|c|}{ Matriz de factores rotada } \\
\hline \multicolumn{4}{|c|}{ Factores } \\
\hline & 1 & 2 & 3 \\
\hline $\begin{array}{l}\text { Q29 Implementación y uso de estándares } \\
\text { nacionales e internacionales. }\end{array}$ & $\mathbf{0 , 8 6 0}$ & & \\
\hline Q30 Logística de la prestación de los servicios. & $\mathbf{0 , 7 1 1}$ & & \\
\hline $\begin{array}{l}\text { Q31 Integración de la tecnología biomédica con las } \\
\text { tecnologías de la información y la comunicación. }\end{array}$ & 0,685 & & \\
\hline Q35 Gestión del conocimiento & $\mathbf{0 , 5 5 9}$ & & \\
\hline Q39 Logística en la cadena de suministro. & $\mathbf{0 , 8 2 7}$ & & \\
\hline Q28 Sistemas de información e informática. & & $\mathbf{0 , 7 7 7}$ & \\
\hline Q36 Integración de la cadena de suministro. & & 0,728 & \\
\hline Q37 Generación de alianzas estratégicas. & & $\mathbf{0 , 8 1 9}$ & \\
\hline $\begin{array}{l}\text { Q38 Implementación y uso de TI (Tecnologías de } \\
\text { la información). }\end{array}$ & & $\mathbf{0 , 8 7 2}$ & \\
\hline Q40 Gestión de la información. & & $\mathbf{0 , 5 8 1}$ & \\
\hline $\begin{array}{l}\text { Q32 Profesionales y especialistas en salud (talento } \\
\text { humano). }\end{array}$ & & & 0,652 \\
\hline Q33 Educación continua y formación. & & & $\mathbf{0 , 8 1 0}$ \\
\hline Q34 Sistemas de incentivos. & & & 0,637 \\
\hline
\end{tabular}

Fuente: Elaboración propia. 


\section{AFE y fiabilidad para la dimensión ¿dónde?}

Inicialmente se validó la aplicabilidad del AFE ya que se encontró un KMO entre 0,5 y 1; y la prueba de esfericidad de Barlett arrojó un resultado menor a 0,05 (Montoya, 2007).

La Tabla 10 presenta los autovalores iniciales para la dimensión “¿dónde?”, se definen cuatro factores de extracción que tienen valores mayores a 1 y explican el $70,83 \%$ de la varianza total de los datos originales.

Tabla 10. Autovalores iniciales para la dimensión "¿dónde?"

\begin{tabular}{|c|c|c|c|}
\hline \multirow{2}{*}{ Ítem } & \multicolumn{3}{|c|}{ Autovalores iniciales } \\
\cline { 2 - 4 } & Total & \% de varianza & \% acumulado \\
\hline 1 & $\mathbf{4 , 3 4 8}$ & 39,523 & 39,523 \\
\hline 2 & $\mathbf{1 , 2 9 7}$ & 11,791 & 51,315 \\
\hline 3 & $\mathbf{1 , 1 2 4}$ & 10,215 & 61,529 \\
\hline 4 & $\mathbf{1 , 0 2 3}$ & 9,296 & $\mathbf{7 0 , 8 2 5}$ \\
\hline 5 & 0,836 & 7,604 & 78,430 \\
\hline 6 & 0,650 & 5,906 & 84,336 \\
\hline 7 & 0,502 & 4,566 & 98,902 \\
\hline 8 & 0,427 & 3,885 & 96,787 \\
\hline 9 & 0,415 & 3,771 & 98,826 \\
\hline 10 & 0,249 & 2,268 & 100,000 \\
\hline 11 & 0,129 & 1,174 & 958 \\
\hline
\end{tabular}

Fuente: Elaboración propia.

Sin embargo, inicialmente se habían planteado de acuerdo con el Radar de la innovación, 3 criterios o factores que son: presencia, conexiones y marca; por esto se decide aplicar el análisis de fiabilidad para determinar si se requiere el cambio de algún ítem y, por ende, de algún factor.

El alfa de Cronbach para esta dimensión fue de 0,81 pero este valor aumentó a 0,86 al eliminar los ítems Q42 y Q48.

La Tabla 11 presenta los resultados de pruebas KMO y esfericidad de Barlett que confirman nuevamente la aplicabilidad del AFE, con el instrumento dimensional modificado y aplicando la rotación Varimax, los factores disminuyen a dos y explican el 60,62\% de la varianza total; la distribución de ítems se presenta en la Tabla 11. 
Tabla 11. Matriz de factores para la dimensión "¿dónde?".

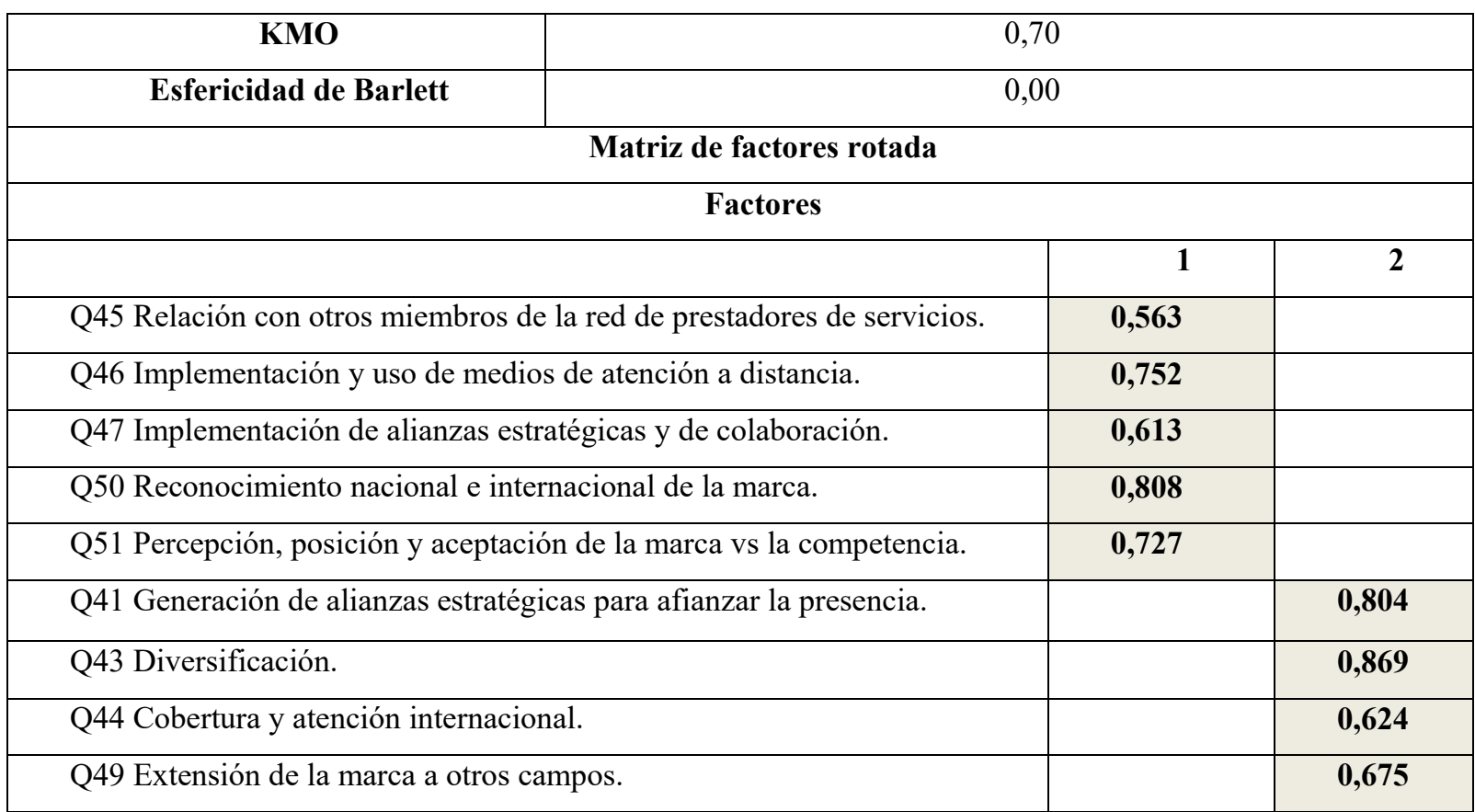

Fuente: Elaboración propia.

\section{Conclusiones.}

En este trabajo se ha sometido a estudio la calidad del instrumento HCI. Como resultado se obtiene un instrumento simplificado, representado en las cuatro dimensiones que propone el Radar de la innovación, con 13 factores o criterios (uno más que el instrumento original) y un total de 43 ítems (6 menos que el instrumento original).

Schramm et al. (2008, p.2) afirman que la "la medición de la innovación está en la infancia" y crece la necesidad de establecer mecanismos que faciliten este proceso pues los directivos de las empresas requieren justificar las inversiones en $\mathrm{I}+\mathrm{D}$ con el fin de mejorar la eficiencia de los recursos y estimar nuevas inversiones para el crecimiento de las empresas.

Las empresas invierten en innovación con el objetivo de ganar participación en el mercado, reducir costos o en un sentido más amplio, mejorar su productividad (OCDE, 2010); la medición del impacto de los resultados de estas inversiones constituye un elemento de gran utilidad para la toma de decisiones en políticas tecnológicas, aseguramiento de recursos, desarrollo de infraestructura, establecimiento de prioridades, entre otras actividades de interés (Torres, Ramos, Lizazo, Monteagudo \& Noda, 2008).

El instrumento desarrollado permite medir el impacto de los 12 criterios del Radar de la innovación en las instituciones prestadoras de servicios de salud, de forma rápida, de bajo costo y teniendo en cuenta todas las áreas y procesos de las entidades.

La principal limitación de esta investigación está relacionada con la muestra de estudio, que aunque es representativa para los expertos en innovación en salud, para el AFE se sugieren muestras de gran tamaño para disminuir la probabilidad de que las correlaciones varíen de una muestra a otra; generalmente se recomienda un tamaño de muestra mínimo de 50; por esta razón las conclusiones obtenidas en este trabajo son significativas para la prueba aplicada (Lloret-Segura, Ferreres-Traver, Hernández-Baeza, \& Tomás-Marco, 2014). 
Los expertos contribuyeron en buena medida a que se diera la concreción del instrumento y por esto se tomaron las decisiones sobre el comportamiento de los criterios, pero se recomienda una validación con muestras más grandes.

El instrumento desarrollado constituye un acercamiento a una herramienta sencilla de medición de la innovación en las instituciones prestadoras de servicios de salud; sin embargo, debe ser sometido a una prueba en por lo menos una institución de salud para evaluar su aplicabilidad.

\section{Recomendaciones.}

Este artículo presenta el desarrollo y validación de una herramienta para caracterizar la innovación en las instituciones prestadoras de servicios de salud a partir de las cuatro dimensiones esenciales del Radar de la innovación.

Para la dimensión “¿qué?” se propone un nuevo modelo factorial al eliminar el ítem Q6 (generación de paquetes de servicios y productos), debido a que los cuatro factores explican el 74,30\% de la varianza total (valor superior al explicado por el modelo inicial); además la confiabilidad de la dimensión es "alta" representada por un alfa de Cronbach de 0,77.

Para la dimensión "¿a quién?" de la HCI, el nuevo modelo factorial se propone a partir de la eliminación de los ítems Q22 y Q25 debido a que los cuatro factores generados explican mejor la varianza total y la confiabilidad de la dimensión es "muy alta" representada por un coeficiente alfa de Cronbach de 0,85 .

La dimensión “¿cómo?” no se modificó debido a que al eliminar los ítems que aumentaban la fiabilidad de la dimensión, la nueva estructura factorial solo explicaría el $64,96 \%$ de la varianza total, valor menor al explicado por los factores iniciales.

En la dimensión “¿dónde?”, la nueva estructura factorial explica el 60,63\% de la varianza total; este valor es inferior al explicado por la estructura factorial inicial, sin embargo en el primer modelo uno de los factores incluía solamente un ítem; el análisis de fiabilidad para esta dimensión propuso entonces la eliminación de los ítems Q42 (ubicación de la institución) y Q48 (confidencialidad de la información y confiabilidad de la institución) pues aumentan la fiabilidad del instrumento para esta dimensión. El modelo factorial propone entonces nuevos factores para cada dimensión en función de la herramienta reducida presentada en la Tabla 12.

Según la validación con los expertos, la eliminación de los ítems Q6 y Q22 se debe a que se desvinculan de la razón de ser del tipo de instituciones analizadas, pues implican ampliar la oferta con actividades diferentes a la prestación de los servicios en salud.

La eliminación de ítems relacionados con la ubicación y la confidencialidad de la información, se debe a que no constituyen una innovación para las instituciones de salud, pues para el primer ítem se estarían privilegiando solo las instituciones que cumplen con ciertas características de localización física lo que les da ventajas competitivas y para el segundo ítem es un tema normativo.

En el nuevo modelo, los factores que agruparon ítems similares a los presentados en la primera versión de la HCI se conservaron; sin embargo, el modelo propuso la generación de nuevos factores que se nombraron de acuerdo a la dimensión y el tipo de innovación que propone el nuevo orden de los ítems.

Se pueden nombrar los nuevos factores pues pertenecen al mismo constructo teórico propuesto por Sawhney et al. (2007) en cada una de las dimensiones del Radar de la innovación (de Carvalho et al., 2016). 
Tabla 12. HCL, versión ajustada.

\section{DIMENSIÓN: ¿QUÉ?}

\section{FACTOR 1: OFERTA}

Q4 Mejoramiento de los servicios existentes.

Q5 Facilidad de acceso y personalización de la oferta.

Q12 Integración de portafolio.

Q13 Identificación del perfil del cliente para brindar mejorar las soluciones.

\section{FACTOR 2: SOLUCIONES}

Q9 Actividades de docencia e investigación.

Q10 Suministro de diferentes líneas de productos y servicios (incluyendo líneas complementarias).

FACTOR 3: PLATAFORMA

\begin{tabular}{|l}
\hline Q7 Dotación y aprovechamiento de la tecnología biomédica. \\
\hline Q8 Aprovechamiento de la infraestructura física. \\
\hline FACTOR 4: SISTEMA DE SERVICIOS \\
\hline Q3 Apertura de servicios. \\
\hline Q11 Generación de asociaciones y alianzas.
\end{tabular}

\section{DIMENSIÓN: ¿A QUIÉN?}

\section{FACTOR 1: EXPERIENCIA DEL CLIENTE}

Q17 Atención e información al cliente.

Q18 Calidad y seguridad en la prestación de los servicios.

Q20 Servicios de preventa y posventa.

\section{FACTOR 2: CLIENTES}

Q14 Relación entre la calidad y el precio.

Q15 Segmentación de clientes.

Q16 Nuevos nichos de mercado (identificación de necesidades insatisfechas).

Q24 Publicidad.

\section{FACTOR 3: OBTENCIÓN DE VALOR}

Q21 Medios de atención.

Q26 Interacción con proveedores, competidores y aliados.

\section{FACTOR 4: RED}

Q23 Participación el proyectos de impulso al sector.

Q27 Participación en redes y trabajo colaborativo.

\section{DIMENSIÓN: ¿CÓMO?}

\section{FACTOR 1: PROCESOS}

Q29 Implementación y uso de estándares nacionales e internacionales.

Q30 Logística de la prestación de los servicios.

Q31 Integración de la tecnología biomédica con las tecnologías de la información y la comunicación. Q35 Gestión del conocimiento. 


\begin{tabular}{|l|}
\hline Q39 Logística en la cadena de suministro. \\
\hline FACTOR 2: CADENA DE SUMINISTRO \\
\hline Q36 Integración de la cadena de suministro. \\
\hline Q37 Generación de alianzas estratégicas. \\
\hline Q38 Implementación y uso de TI (Tecnologías de la información). \\
\hline Q40 Gestión de la información. \\
\hline Q28 Sistemas de información e informática. \\
\hline Q32 Profesionales y especialistas en salud (talento humano). \\
\hline Q33 Educación continua y formación. \\
\hline Q34 Sistemas de incentivos. \\
\hline \\
\hline Q45 Relación con otros miembros de la red de prestadores de servicios. \\
\hline Q46 Implementación y uso de medios de atención a distancia. \\
\hline Q47 Implementación de alianzas estratégicas y de colaboración. \\
\hline Q50 Reconocimiento nacional e internacional de la marca. \\
\hline Q51 Percepción, posición y aceptación de la marca vs la competencia. \\
\hline \\
\hline Q41 Generación de alianzas estratégicas para afianzar la presencia. \\
\hline Q43 Diversificación. \\
\hline Q44 Cobertura y atención internacional. \\
\hline Q49 Extensión de la marca a otros campos. \\
\hline
\end{tabular}

Fuente: Elaboración propia.

En consecuencia, el instrumento HCI de 43 ítems presentado en la Tabla 12, ofrece mayor calidad que el cuestionario original, tanto a nivel general como para cada una de sus dimensiones específicas.

\section{Referencias}

Babbie, E. (2000). Fundamentos de la investigación social. Mexico: International Thomson.

Barba, E. (2012). Los 10 tipos de innovación. El ejemplo de red bull. Gestionando la innovación. Recuperado de http://www.enricbarba.com/2012/11/los-10-tipos-de-innovacion-el-ejemplo-de-redbull/

Campo-Arias, A., \& Oviedo, H.C. (2008). Propiedades Psicométricas de una Escala: la Consistencia Interna. Revista de Salud Pública, 10(5), 831-839. DOI: 10.1590/S0124-00642008000500015.

Corral, Y. (2009). Validez y confiabilidad de los instrumentos de investigación para la recolección de datos. Ciencias de la Educación, 19(33), 228-247. 
de Carvalho, G.D.G., dos Anjos Almeida, M.C.A., Quandt, C.O., de Carvalho, H.G., Cruz, J.A.W., \& da Veiga, C.P. (2016). Estrutura de Agrupamento das Dimensões do Radar da Inovação de Micro e Pequenas Empresas no Brasil. Revista Espacios, 37(23), 19-25.

del Llano Señarís, J., Marténez-Cantarero, J.F., Gol, J., \& Raigada, F. (2002). Análisis cualitativo de las innovaciones organizativas en hospitales públicos españoles. Gaceta sanitaria / S.E.S.P.A.S, 16(5), 408-416. DOI: 10.1016/S0213-9111(02)71950-X.

Detrinidad, E. (2016). Análisis Factorial Exploratorio y Confirmatorio aplicado al modelo de secularización propuesto por Inglehart-Norris. Periodo 2010-2014 (Estudio de caso España, Estados Unidos, Alemania, Holanda) WSV. Universidad de Granada.

Djellal, F., \& Gallouj, F. (2005). Mapping innovation dynamics in hospitals. Research Policy, 34(6), 817-835. DOI: 10.1016/j.respol.2005.04.007.

Giacometti-Rojas, L.F. (2013). Innovación tecnológica y desarrollo de ventaja competitiva en la atención a la salud: enfoque conceptual y metodológico. Revista gerencia y políticas de salud, 12(25), 66-82. DOI: 10.11144/Javeriana.rgsp12-25.itdv.

Guan, J.C., Yam, R.C.M., Mok, C.K., \& Ma, N. (2006). A study of the relationship between competitiveness and technological innovation capability based on DEA models. European Journal of Operational Research, 170(3), 971-986. DOI: 10.1016/j.ejor.2004.07.054.

Hernández-Sampieri, R., Fernández, C., \& Baptista, M. del P. (2010). Metodología de la investigación. México: Mcgraw-Hill

Huh, J., DeLorme, D.E., \& Reid, L. N. (2006). Perceived Third-Person Effects and Consumer Attitudes on Prevetting and Banning DTC Advertising. The journal of Consumer Affairs, 40(1), 90-116. DOI: 10.1111/j.1745-6606.2006.00047.x.

Hyrkäs, K., Appelqvist-Schmidlechner, K., \& Oksa, L. (2003). Validating an instrument for clinical supervision using an expert panel. International Journal of Nursing Studies, 40(6), 619-625. DOI: 10.1016/S0020-7489(03)00036-1.

Jaramillo, S., \& Osses, S. (2012). Validación de un Instrumento sobre Metacognición para Estudiantes de Segundo Ciclo de Educación General Básica. Estudios pedagógicos (Valdivia), 38(2), 117-131. DOI: $10.4067 /$ S0718-07052012000200008

Jaramillo, H., Latorre, C., Albán, C., \& Lopera, C. (2008). El hospital como organización de conocimiento y espacio de investigación y formación: los recursos humanos en salud y su tránsito a comunidades científicas: el caso de la investigación clínica en Colombia. Editorial Universidad del Rosario. Recuperado de https://www.jstor.org/stable/j.ctt1b18wpz.

Kaplan, R.M., Saccuzzo, D.P., \& Reyes, M. de L. (2006). Pruebas psicológicas: principios, aplicaciones y temas. Mexico: Thomson.

Keeley, L., Pikkel, R., Quinn, B., \& Walters, H. (2013). Ten types of innovation. The building blocks of breakthroughs. USA: Willey.

Länsisalmi, H., Kivimäki, M., Aalto, P., \& Ruoranen, R. (2006). Innovation in Healthcare: A Systematic Review of Recent Research. Nursing Science Quarterly, 19(1), 66-72. DOI: $10.1177 / 0894318405284129$. 
Lloret-Segura, S., Ferreres-Traver, A., Hernández-Baeza, A., \& Tomás-Marco, I. (2014). El análisis factorial exploratorio de los ítems: Una guía práctica, revisada y actualizada. Anales de Psicologia, 30(3), 1151-1169. DOI: 10.6018/analesps.30.3.199361.

Montoya, O. (2007). Aplicación del análisis factorial a la investigación de mercados. Caso de estudio. Scientia et technica, 1(35), 281-286. Recuperado de http://revistas.utp.edu.co/index.php/revistaciencia/article/view/5443

OCDE (2005). Manual de Oslo: Guía para la recogida e interpretación de datos sobre innovación. EUROSTAT Y OCDE. DOI: 10.1787/9789264065659-es.

OCDE (2010). La medición de la innovación: una nueva perspectiva. Recuperado de http://www.foroconsultivo.org.mx/libros_editados/medicion_innovacion.pdf

OPS (2009). Innovación para la Salud Pública en las Américas: Promoción de la investigación y el desarrollo de productos para la salud. Pananá: Organización Panamericana de la Salud.

Palencia-Sánchez, F., \& García-Ubaque, J. C. (2016). Innovación e investigación en hospitales universitarios. Revista Facultad de Medicina, 64(4), 741-747. DOI: 10.15446/revfacmed.v64n4.54837.

Pérez-Gil, J.A., Chacón, S., \& Moreno, R. (2000). Validez de constructo: El uso de análisis factorial exploratorio-confirmatorio para obtener evidencias de validez. Psicothema, 12(SUPPL. 2), 442-446. DOI: $10.1177 / 0013164412473825$.

Pérez, E.R., \& Medrano, L. (2010). Análisis factorial exploratorio: Bases conceptuales y metodológicas. Revista Argentina de Ciencias del Comportamiento, 2(1889), 58-66. DOI: 10.2307/2183556.

Ruiz, C. (1998). Instrumentos de investigación educativa: un enfoque cuantitativo y cualitativo para la recolección y análisis de datos. Houston: DANAGA Training and Consulting.

Sawhney, M., Wolcott, R., \& Arroniz, I. (2007). The 12 different ways for companies to innovate. IEEE Engineering Management Review, 35(1), 45-45. DOI: 10.1109/EMR.2007.329139.

Schramm, C.J., Arora, A., Chandy, R.K., Cooper, K., Jorgenson, D.W., Siegel, D.S.,... Menzer, J. (2008). Innovation Measurement: Tracking the State of Innovation in the American Economy. USA: Secretary of commerce, U.S. Department of Commerce. DOI: 10.2139/ssrn.1090630.

Skjong, R., \& Wentworth, B. (2000). Expert Judgement and Risk Perception. Det Norske Veritas, 1-8. Recuperado de http://research.dnv.com/skj/Papers/SkjWen.pdf

Thune, T., \& Mina, A. (2016). Hospitals as innovators in the health-care system: A literature review and research agenda. Research Policy, 45(8), 1545-1557. DOI: 10.1016/j.respol.2016.03.010.

Torres, V., Ramos, N., Lizazo, D., Monteagudo, F., \& Noda, A. (2008). Modelo estadístico para la medición del impacto de la innovación o transferencia tecnológica en la rama agropecuaria. Revista Cubana de Ciencia Agrícola, 42(2), 133-139.

Zamora, S., Monroy, L., \& Chávez, C. (2009). Análisis factorial: una técnica para evaluar la dimensionalidad de las pruebas. Papeles de Psicólogo, 31(1), 18-33. 